\title{
On-line Update Method for the Sensitivity Consistency Model of Interconnected Power Grid Based on Electric Power Big Data
}

\author{
Shuai Lian ${ }^{*}$, Bintang $\mathrm{Li}^{2}$, Jianbo Wang ${ }^{3}$ and Rui Jiang ${ }^{4}$ \\ ${ }^{1} 713$ Research Institute of China State Shipbuilding Corporation, Zheng Zhou, He Nan, China \\ ${ }^{2}$ State Grid Qinghai Electric Power Company Maintenance Company, Xi Ning, Qing Hai, China \\ ${ }^{3}$ State Grid Zhejiang Electric Power Co., Ltd. Taizhou Lu Qiao District Power Supply Company, Lu Qiao, Tai Zhou, Zhe Jiang, China \\ ${ }^{4}$ School of Electrical Engineering and Automation, Hefei University of Technology, He Fei, An Hui, China
}

\begin{abstract}
Real-time fast calculation of the power flow of the interconnected power grid is an important guarantee for the reliable operation of the interconnected power grid. The topology of the interconnected power grid is complex, and the calculation of the power flow of the whole network is large and timeconsuming. The sensitivity equivalent model can effectively simplify the interconnected power grid and shorten the time of the power flow calculation of the whole network. The operating state of the power grid is constantly changing. In order to ensure the accuracy of the power flow calculation results, it is necessary to update the uniform sensitivity equivalent model in real time. Due to factors such as the vertical management system between the interconnected power grids and the principle of commercial confidentiality, it is difficult to share information between interconnected power grids in real time, and the sensitivity equivalent model cannot be updated in real time, resulting in too much error in the calculation results and no reference value. To solve this problem, this paper proposes an online update method for the sensitivity equivalent model of the interconnected power grid based on power big data to solve the problem of excessive power flow calculation errors caused by the untimely update of the equivalent model parameters, and to ensure the operational reliability of the interconnected power grid.
\end{abstract}

\section{Introduction}

With the continuous growth of the world's electric energy consumption and the innovation of high-voltage transmission technology, long-distance and large-scale grid interconnection and power transmission no longer have technical obstacles $[1,2]$. There are many advantages to interconnecting power grids in different regions. It can promote the consumption of renewable energy and reduce the dependence on fossil energy, reduce environmental pollution, improve social benefits, adjust the peak-tovalley difference of the load, and improve the reliability of the power grid. The scale of existing interconnected power grids is getting bigger and bigger. Not only are the power grids interconnected across regions, but also the interconnection of transnational power grids and global transcontinental power grids has also formed a scale $[3,4]$.

While the interconnected power grid brings obvious benefits, it also leads to many problems. The topology is complex and the system is huge. It is difficult for the existing computing power to perform complete power flow calculations on the entire interconnected power grid in real time, and to analyze the weaknesses of the system, which leads to local problems. The risks of the power grid spread throughout the interconnected power grid and have a great impact on the reliability of the power grid.
In view of the problem that the power flow calculation of the entire interconnected power grid cannot be performed quickly, based on the power system topology [5-7], some scholars have proposed a sensitivity consistent model (SC model) [8] to reduce the power flow calculation time. Although this method simplifies the interconnected power grid and improves the efficiency of power flow calculation of the interconnected power grid, the model cannot reflect the ever-changing interconnected power grid, which makes the calculation results of the power flow of the interconnected power grid too large and loses reference value. Therefore, timely updating the equivalent model is the key to improving the results of power flow calculations. However, large-scale interconnected power grids are composed of interconnected networks of different power companies, different regions and even different countries. Due to factors such as management systems and commercial confidentiality, it is difficult to share the operating data of local power grids with all companies on the interconnected power grid in time. This is the main problem faced by online real-time updating of the equivalent model of the interconnected power grid.

In response to this problem, the article proposes an online update method of the sensitivity equivalent model based on the power big data based on the electric power big data recorded by the advanced measurement

${ }^{*}$ Corresponding author's e-mail: 2020001@zzuli.edu.cn 
equipment. The analysis of the examples verifies that this method can effectively solve the problem of the inability to be online in real time due to various commercial factors, and improve the reliability of the power flow calculation.

\section{Sensitivity Consistency Model}

The sensitivity consistency model (SC model) is a static equivalent model that fully considers the characteristics of various electrical components and ensures the sensitivity between the electrical components does not change before and after the equivalent value. In the existing equivalence method, the after-equivalence power flow calculation results of the sensitivity consistency model are basically consistent with the pre-equivalence power flow calculation results. This paper updates the model online based on power big data. The consistent sensitivity model is shown in Figure 1:

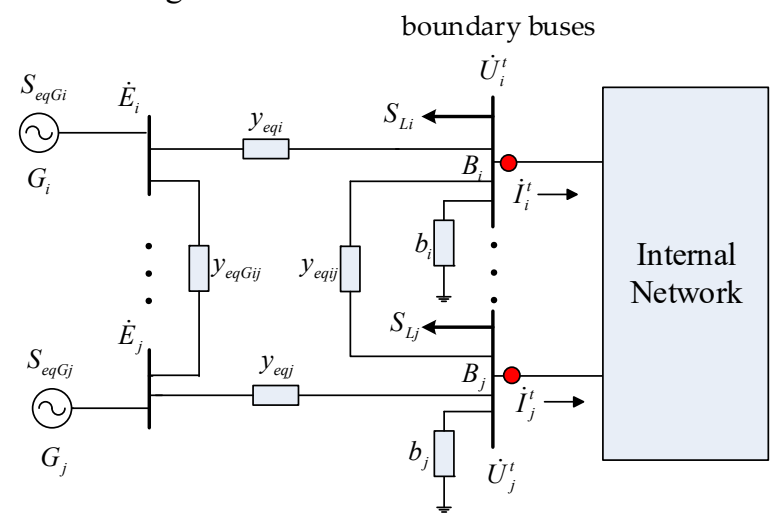

Figure $1 \mathrm{SC}$ equivalent system diagram

The SC model is mainly composed of three kinds of variables:

- Admittance variables: $y_{\text {eqi }}$ and $y_{\text {eqj }}$ are the admittances of equivalent branches between $G_{i}$ and $B_{i}$, and between $G_{j}$ and $B_{j}$, respectively. $y_{e q i j}$ and $y_{\text {eqGij }}$ are the admittances of the equivalent branches between $B_{i}$ and $B_{j}$, and between $G_{i}$ and $G_{j}$, respectively. $b_{i}$ and $b_{j}$ are the susceptances of the equivalent grounding branches connected with the boundary buses $b_{i}$ and $b_{j}$, respectively. where $G_{i}$ and $G_{j}$ are the generator buses, $B_{i}$ and $B_{j}$ are the boundary buses, $i, j=1 \cdots N_{B} i \neq j$, and $N_{B}$ is the number of boundary buses.

- Voltage variable: $\dot{E}_{i}$ and $\dot{E}_{j}$ are the voltage phasors at the equivalent generator buses $G_{i}$ and $G_{j}$, respectively.

- Power variables: $S_{L i}$ and $S_{L j}$ are the equivalent loads at the boundary buses $B_{i}$ and $B_{j}$, respectively. $S_{e q G i}$ and $S_{e q G j}$ are the equivalent generator output powers at $G_{i}$ and $G_{j}$, respectively.

\section{Online Update of the SC Model Based on Electric Power Big Data}

\subsection{Electric Power Big Data}

Due to the management system and the principle of commercial confidentiality, the regional power grids of the interconnected power grid cannot know the network topology and real-time operating status data of the interconnected power grid. With the development of smart grid and information technology, power big data is widely used, and the problem of information isolation can be solved very well based on power big data. Electric power big data [9] is mainly composed of three categories:

Grid operation, equipment detection and status monitoring: included in energy management system, distribution network management system, wide area measurement system, fault measurement system, power grid dispatching system, etc.;

- $\quad$ Electricity marketing data: It mainly includes marketing business system, electric energy metering system, electricity consumption information collection system, etc.

- Power grid management data: included in collaborative office systems, enterprise resource planning systems (ERP), etc.

Based on the data measured by the synchronous vector measurement device (PMU) in the wide area measurement system, we can online update the SC model.

\subsection{Measurement Equations}

As shown in Figure 1, the parameters to be estimated for the SC model are $x=\left\{\dot{E}_{i}, \dot{E}_{j}, y_{\text {eqi }}, y_{\text {eqj }}, y_{\text {eqij }}, b_{i}\right.$, $\left.b_{j}, S_{L i}, S_{L j}, S_{e q G i}, S_{e q G j}\right\}$. The boundary PMU measures the voltage and current of bus $B_{i}$ as $\dot{U}_{i}^{t}$ and $\dot{I}_{i}^{t}$, and bus $B_{j}$ as $\dot{U}_{j}^{t}$ and $\dot{I}_{j}^{t}$, respectively. where, $i, j=1 \cdots N_{B}, \quad i \neq j, N_{B}$ is the number of boundary buses, $t=1, \cdots, M, \quad M$ is the number of PMU samples in the given period of time. The following measurement equations can be derived from Kirchhoff's current law:

$$
f_{i}^{t}(x)=\left(\dot{E}_{i}-\dot{U}_{i}^{t}\right) y_{e q i}-\dot{I}_{i}^{t}-\left(\frac{S_{L i}}{\dot{U}_{i}^{t}}\right)^{*}-b_{i} \dot{U}_{i}^{t}-\sum_{i=1, j \neq i}^{N_{B}}\left(\dot{U}_{i}^{t}-\dot{U}_{j}^{t}\right) y_{e q i j}=0
$$




$$
g_{i}^{t}(x)=\left(\frac{S_{e q G i}}{\dot{E}_{i}}\right)^{*}-\sum_{i=1, i \neq j}^{N_{B}}\left(\dot{E}_{i}-\dot{E}_{j}\right) y_{e q G i j}-\left(\dot{E}_{i}-\dot{U}_{i}^{t}\right) y_{e q i}=0
$$

The complex variables in Equations (1) and (2) are the real part, and Formulas (3)-(6) are obtained as follows: respectively expanded according to the imaginary part and

$$
\begin{aligned}
& f_{i(\mathrm{Re})}^{t}(x)=\left(\dot{E}_{i}-\dot{U}_{i}^{t}\right)_{\mathrm{Re}} y_{\text {eqq }(\mathrm{Re})}\left(\dot{U}_{i}^{t}\right)_{\mathrm{Re}}^{*}+\left(\dot{E}_{i}-\dot{U}_{i}^{t}\right)_{\mathrm{Im}} y_{\text {eqi }(\mathrm{Im})}\left(\dot{U}_{i}^{t}\right)_{\mathrm{Re}}^{*} \\
& +\left(\dot{E}_{i}-\dot{U}_{i}^{t}\right)_{\mathrm{Re}} y_{\text {eqi(Im) }}\left(\dot{U}_{i}^{t}\right)_{\mathrm{Im}}^{*}+\left(\dot{E}_{i}-\dot{U}_{i}^{t}\right)_{\mathrm{Im}} y_{\text {eqi }(\mathrm{Re})}\left(\dot{U}_{i}^{t}\right)_{\mathrm{Im}}^{*}-\left(\dot{U}_{i}^{t}\right)_{\mathrm{Re}}^{*} \dot{I}_{i(\mathrm{Re})}^{t} \\
& -\left(\dot{U}_{i}^{t}\right)_{\mathrm{Im}}^{*} \dot{I}_{i(\mathrm{~mm})}^{t}-\left(S_{L i}\right)_{\mathrm{Re}}^{*}-\sum_{i=1, i \neq j}^{N_{B}}\left[\left(\dot{U}_{i}^{t}-\dot{U}_{j}^{t}\right)_{\mathrm{Re}} y_{\text {eqjij(Re) }}\left(\dot{U}_{i}^{t}\right)_{\mathrm{Re}}^{*}\right. \\
& +\left(\dot{U}_{i}^{t}-\dot{U}_{j}^{t}\right)_{\mathrm{Re}} y_{\text {eqij }(\mathrm{mm})}\left(\dot{U}_{i}^{t}\right)_{\mathrm{Im}}^{*}+\left(\dot{U}_{i}^{t}-\dot{U}_{j}^{t}\right)_{\mathrm{Im}} y_{\text {eqij }(\mathrm{Im})}\left(\dot{U}_{i}^{t}\right)_{\mathrm{Re}}^{*} \\
& \left.+\left(\dot{U}_{i}^{t}-\dot{U}_{j}^{t}\right)_{\mathrm{Im}} y_{\text {eqij }(\mathrm{Re})}\left(\dot{U}_{i}^{t}\right)_{\mathrm{Im}}^{*}\right]=0 \\
& f_{i(\mathrm{Im})}^{t}(x)=\left(\dot{E}_{i}-\dot{U}_{i}^{t}\right)_{\mathrm{Im}} y_{\text {eqi(Re) }}\left(\dot{U}_{i}^{t}\right)_{\mathrm{Re}}^{*}+\left(\dot{E}_{i}-\dot{U}_{i}^{t}\right)_{\mathrm{Im}} y_{\text {eqi }(\mathrm{Im})}\left(\dot{U}_{i}^{t}\right)_{\mathrm{Im}}^{*} \\
& +\left(\dot{E}_{i}-\dot{U}_{i}^{t}\right)_{\mathrm{Re}} y_{\text {eqi(Re) }}\left(\dot{U}_{i}^{t}\right)_{\mathrm{Im}}^{*}+\left(\dot{E}_{i}-\dot{U}_{i}^{t}\right)_{\mathrm{Re}} y_{\text {eqi(Im) }}\left(\dot{U}_{i}^{t}\right)_{\mathrm{Re}}^{*}-\left(\dot{U}_{i}^{t}\right)_{\mathrm{Re}}^{*} \dot{I}_{i(\mathrm{Im})}^{t} \\
& -\left(\dot{U}_{i}^{t}\right)_{\mathrm{Im}}^{*} \dot{I}_{i(\mathrm{Re})}^{t}-\left(S_{L i}\right)_{\mathrm{Im}}^{*}-\sum_{i=1, i \neq j}^{N_{B}}\left[\left(\dot{U}_{i}^{t}-\dot{U}_{j}^{t}\right)_{\mathrm{Re}} y_{\text {eqji }(\mathrm{Re})}\left(\dot{U}_{i}^{t}\right)_{\mathrm{Im}}^{*}\right. \\
& +\left(\dot{U}_{i}^{t}-\dot{U}_{j}^{t}\right)_{\mathrm{Im}} y_{\text {eqij }(\mathrm{mm})}\left(\dot{U}_{i}^{t}\right)_{\mathrm{Im}}^{*}+\left(\dot{U}_{i}^{t}-\dot{U}_{j}^{t}\right)_{\mathrm{Re}} y_{\text {eqji }(\mathrm{Im})}\left(\dot{U}_{i}^{t}\right)_{\mathrm{Re}}^{*} \\
& \left.+\left(\dot{U}_{i}^{t}-\dot{U}_{j}^{t}\right)_{\mathrm{Im}} y_{\text {eqij }(\mathrm{Re})}\left(\dot{U}_{i}^{t}\right)_{\mathrm{Re}}^{*}\right]-b_{i} \dot{U}_{i}^{t}\left(\dot{U}_{i}^{t}\right)^{*}=0 \\
& g_{i(\mathrm{Re})}^{t}(x)=\left(S_{e q G i}\right)_{\mathrm{Re}}^{*}-\sum_{i=1, i \neq j}^{N_{B}}\left[\left(\dot{E}_{i}-\dot{E}_{j}\right)_{\mathrm{Re}} y_{e q G i j(\mathrm{Re})}\left(\dot{E}_{i}\right)_{\mathrm{Re}}^{*}\right. \\
& +\left(\dot{E}_{i}-\dot{E}_{j}\right)_{\mathrm{Re}} y_{\text {eqGij }(\mathrm{Im})}\left(\dot{E}_{i}\right)_{\mathrm{Im}}^{*}+\left(\dot{E}_{i}-\dot{E}_{j}\right)_{\mathrm{Im}} y_{\text {eqGij }(\mathrm{Im})}\left(\dot{E}_{i}\right)_{\mathrm{Re}}^{*} \\
& \left.+\left(\dot{E}_{i}-\dot{E}_{j}\right)_{\mathrm{Im}} y_{e q G i j(\mathrm{Re})}\left(\dot{E}_{i}\right)_{\mathrm{Im}}^{*}\right]-\left(\dot{E}_{i}-\dot{U}_{i}^{t}\right)_{\mathrm{Re}} y_{e q i(\mathrm{Re})}\left(\dot{E}_{i}\right)_{\mathrm{Re}}^{*} \\
& -\left(\dot{E}_{i}-\dot{U}_{i}^{t}\right)_{\mathrm{Re}} y_{\text {eqi }(\mathrm{Im})}\left(\dot{E}_{i}\right)_{\mathrm{Im}}^{*}-\left(\dot{E}_{i}-\dot{U}_{i}^{t}\right)_{\mathrm{Im}} y_{\text {eqi }(\mathrm{Im})}\left(\dot{E}_{i}\right)_{\mathrm{Re}}^{*} \\
& -\left(\dot{E}_{i}-\dot{U}_{i}^{t}\right)_{\mathrm{Im}} y_{\text {eqi(Re) }}\left(\dot{E}_{i}\right)_{\mathrm{Im}}^{*}=0 \\
& g_{i(\mathrm{Im})}^{t}(x)=\left(S_{e q G i}\right)_{\mathrm{Im}}^{*}-\sum_{i=1, i \neq j}^{N_{B}}\left[\left(\dot{E}_{i}-\dot{E}_{j}\right)_{\mathrm{Im}} y_{e q G i j(\mathrm{Re})}\left(\dot{E}_{i}\right)_{\mathrm{Re}}^{*}\right. \\
& +\left(\dot{E}_{i}-\dot{E}_{j}\right)_{\mathrm{Im}} y_{\text {eqGij }(\mathrm{Im})}\left(\dot{E}_{i}\right)_{\mathrm{Im}}^{*}+\left(\dot{E}_{i}-\dot{E}_{j}\right)_{\mathrm{Re}} y_{e q G i j(\mathrm{Im})}\left(\dot{E}_{i}\right)_{\mathrm{Re}}^{*} \\
& \left.+\left(\dot{E}_{i}-\dot{E}_{j}\right)_{\mathrm{Re}} y_{e q G i j(\mathrm{Re})}\left(\dot{E}_{i}\right)_{\mathrm{Im}}^{*}\right]-\left(\dot{E}_{i}-\dot{U}_{i}^{t}\right)_{\mathrm{Im}} y_{e q i(\mathrm{Re})}\left(\dot{E}_{i}\right)_{\mathrm{Re}}^{*} \\
& -\left(\dot{E}_{i}-\dot{U}_{i}^{t}\right)_{\mathrm{Im}} y_{\text {eqi }(\mathrm{Im})}\left(\dot{E}_{i}\right)_{\mathrm{Im}}^{*}-\left(\dot{E}_{i}-\dot{U}_{i}^{t}\right)_{\mathrm{Re}} y_{\text {eqi }(\mathrm{Im})}\left(\dot{E}_{i}\right)_{\mathrm{Re}}^{*} \\
& -\left(\dot{E}_{i}-\dot{U}_{i}^{t}\right)_{\mathrm{Re}} y_{\text {eqi }(\mathrm{Re})}\left(\dot{E}_{i}\right)_{\mathrm{Im}}^{*}=0
\end{aligned}
$$

Where ()$^{*}$ denotes the conjugate operation. The subscripts $(*)_{\operatorname{Re}}$ and $(*)_{\operatorname{Im}}$ in the Equations (3)-(6) respectively represent the real part and the imaginary parts of the complex variable $(*) . f_{i(\mathrm{Re})}^{t}(x), f_{i(\operatorname{Im})}^{t}(x)$, $g_{i(\mathrm{Re})}^{t}(x)$ and $g_{i(\mathrm{Im})}^{t}(x)$ are the measurement equations separating the real and imaginary parts of (1) and (2), respectively. Through the above measurement equation, with the inspecting data $\dot{U}_{i}^{t}, \dot{I}_{i}^{t}, \dot{U}_{j}^{t}$ and $\dot{I}_{j}^{t}$ of the boundary PMU, all SC model parameters $x$

$$
L=\min \left\{\sum_{t=1}^{M} \sum_{i=1}^{N_{B}}\left[\left(f_{i(\mathrm{Re})}^{t}(x)\right)^{2}+\left(f_{i(\mathrm{Im})}^{t}(x)\right)^{2}+\left(g_{i(\mathrm{Re})}^{t}(x)\right)^{2}+\left(g_{i(\mathrm{Im})}^{t}(x)\right)^{2}\right]\right\}
$$

In order to ensure the accuracy of the solution, the number of measurement equations must be greater than the number of unknown parameters. From the SC model can be update, where, $x=\left\{\dot{E}_{i}, \dot{E}_{j}, y_{\text {eqi }}, y_{\text {eqj }}, y_{\text {eqij }}\right.$, $\left.b_{i}, b_{j}, S_{L i}, S_{L j}, S_{e q G i}, S_{e q G j}\right\}$.

\subsection{Online Update of the SC Model Based on Electric Power Big Data}

Based on the PMU measurement data and the established measurement formula, the least square method is used to update the sensitivity consistency model, and the updated model is shown in formula (7): shown in Figure 1, we can see that the number of each parameter $\left(\dot{E}_{i}\right)_{\operatorname{Re}},\left(\dot{E}_{i}\right)_{\mathrm{Im}},\left(S_{e q G i}\right)_{\mathrm{Re}},\left(S_{e q G i}\right)_{\mathrm{Im}}$, 
$\left(S_{L i}\right)_{\mathrm{Re}}, \quad\left(S_{L i}\right)_{\mathrm{Im}}, y_{\text {eqi }(\mathrm{Re})}, \quad y_{\text {eqi }(\mathrm{Im})}, \quad b_{i}$ is $N_{B}$ respectively, and there are $9 N_{B}$ unknown parameters together. The number of each parameter $y_{\text {eqij(Re) }}$, $y_{e q i j(\mathrm{Im})}, \quad y_{e q G i j(\mathrm{Re})}, \quad y_{e q G i j(\mathrm{Im})}$ is $N_{B}\left(N_{B}-1\right) / 2$ respectively, and there are $2 N_{B}\left(N_{B}-1\right)$ unknown parameters together. The number of measurement equations is $4 M N_{B}$, which must satisfy the following inequality:
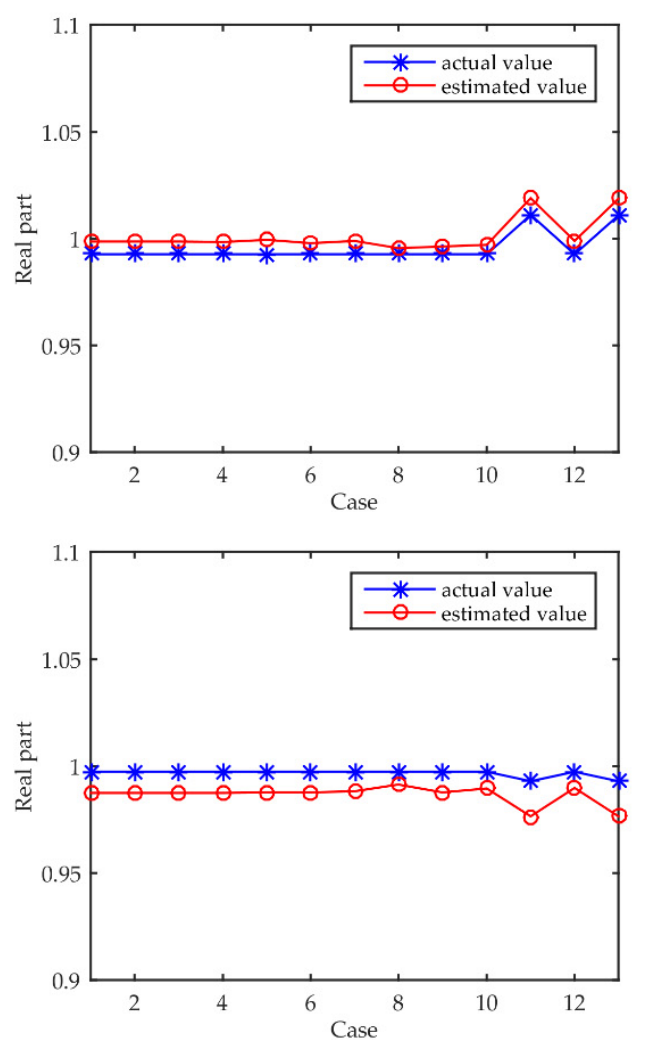

Figure 4 Voltage parameters of the SC model.

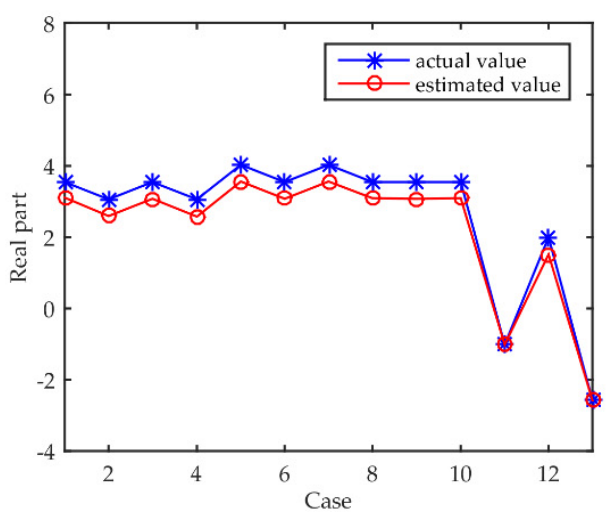

$$
4 M N_{B}>9 N_{B}+2 N_{B}\left(N_{B}-1\right)
$$

After transformation, it can be obtained that:

$$
M>N_{B} / 2+7 / 4
$$

All parameters of the $\mathrm{SC}$ model can be updated through equations (3)-(7).

\section{Results and Discussion}

\subsection{Test System Introduction}
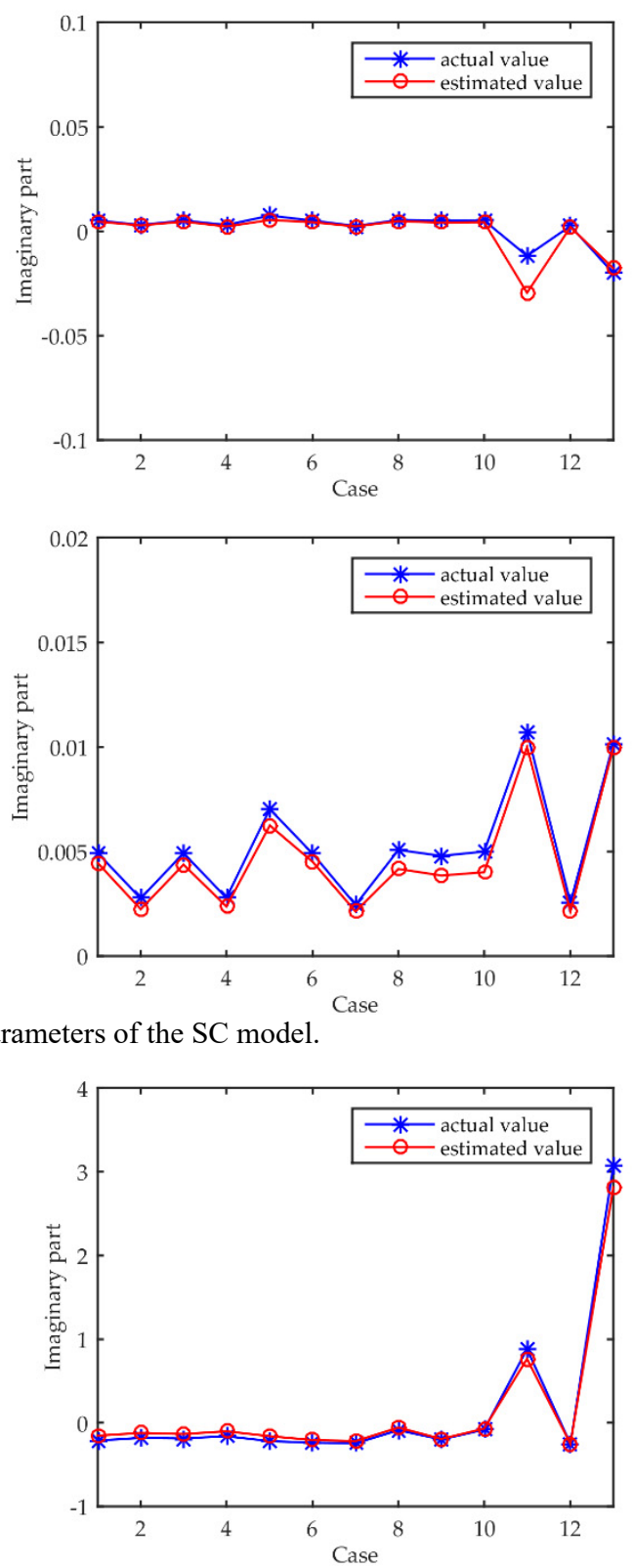

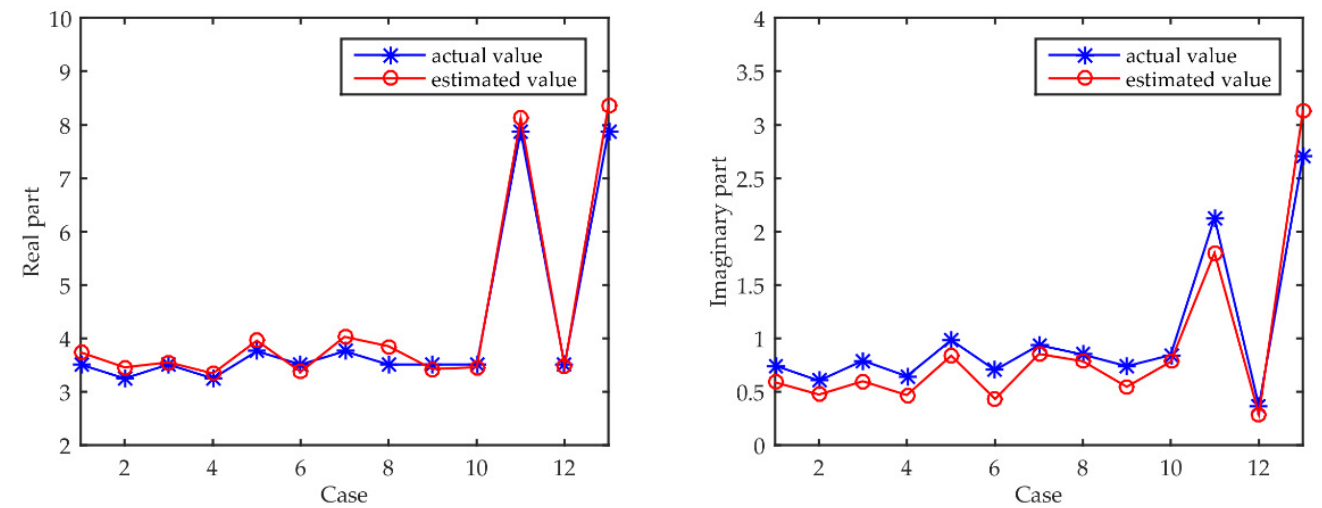

Figure 5 Generator output of the SC model.
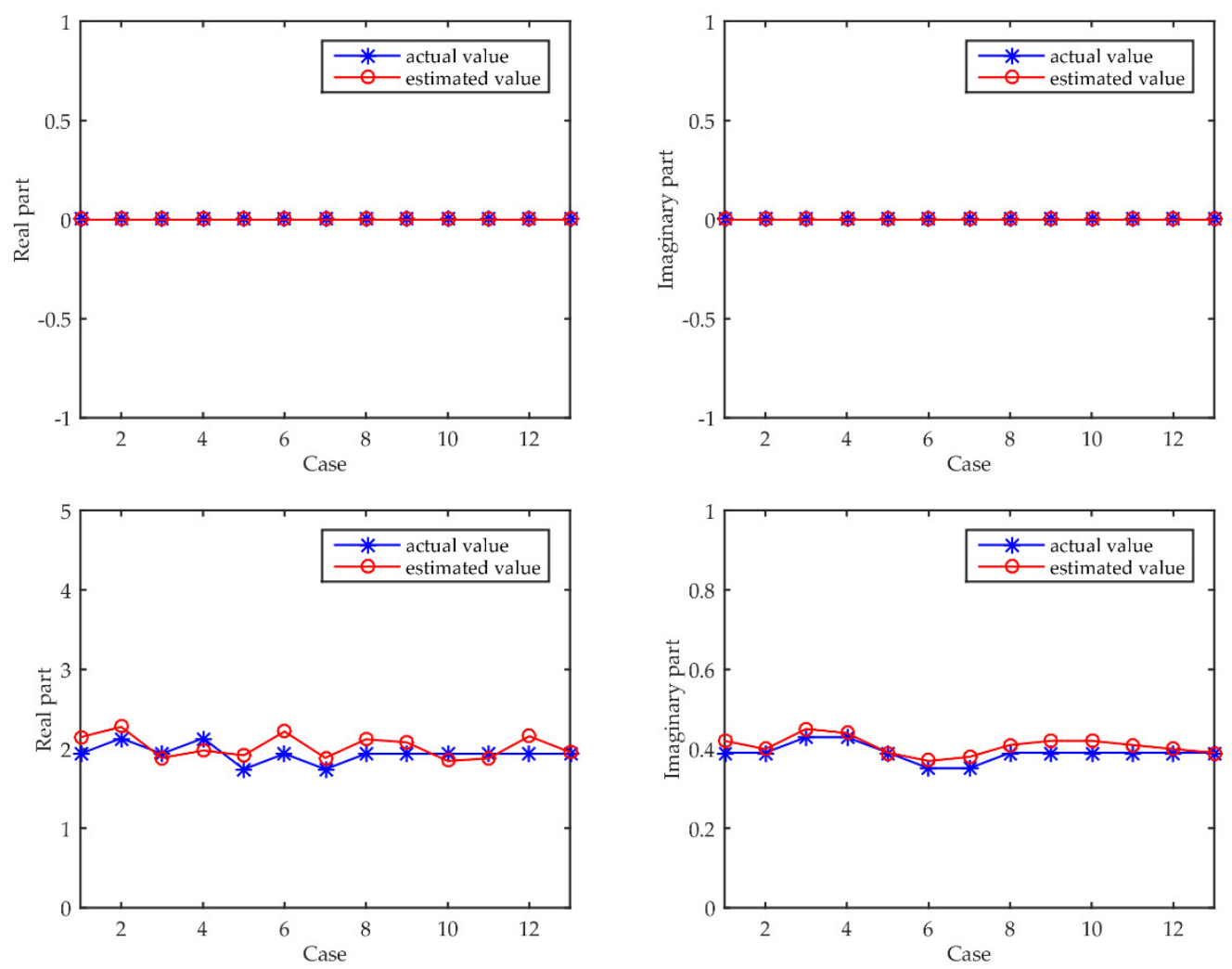

Figure 6 Load parameters of the SC model
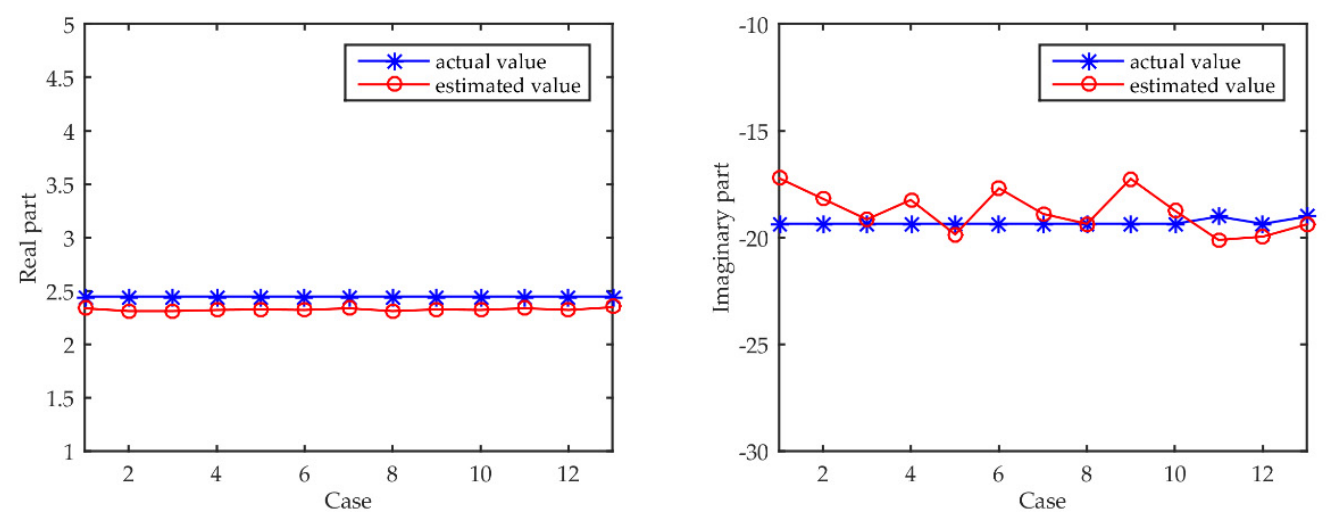

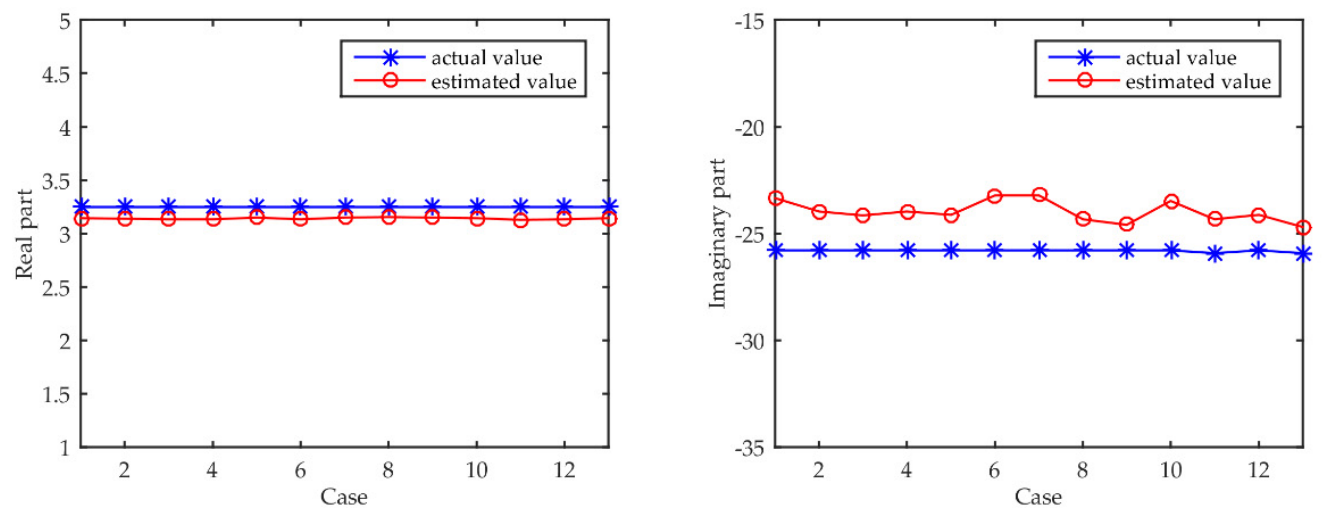

Figure 7 Admittance parameters of the SC model
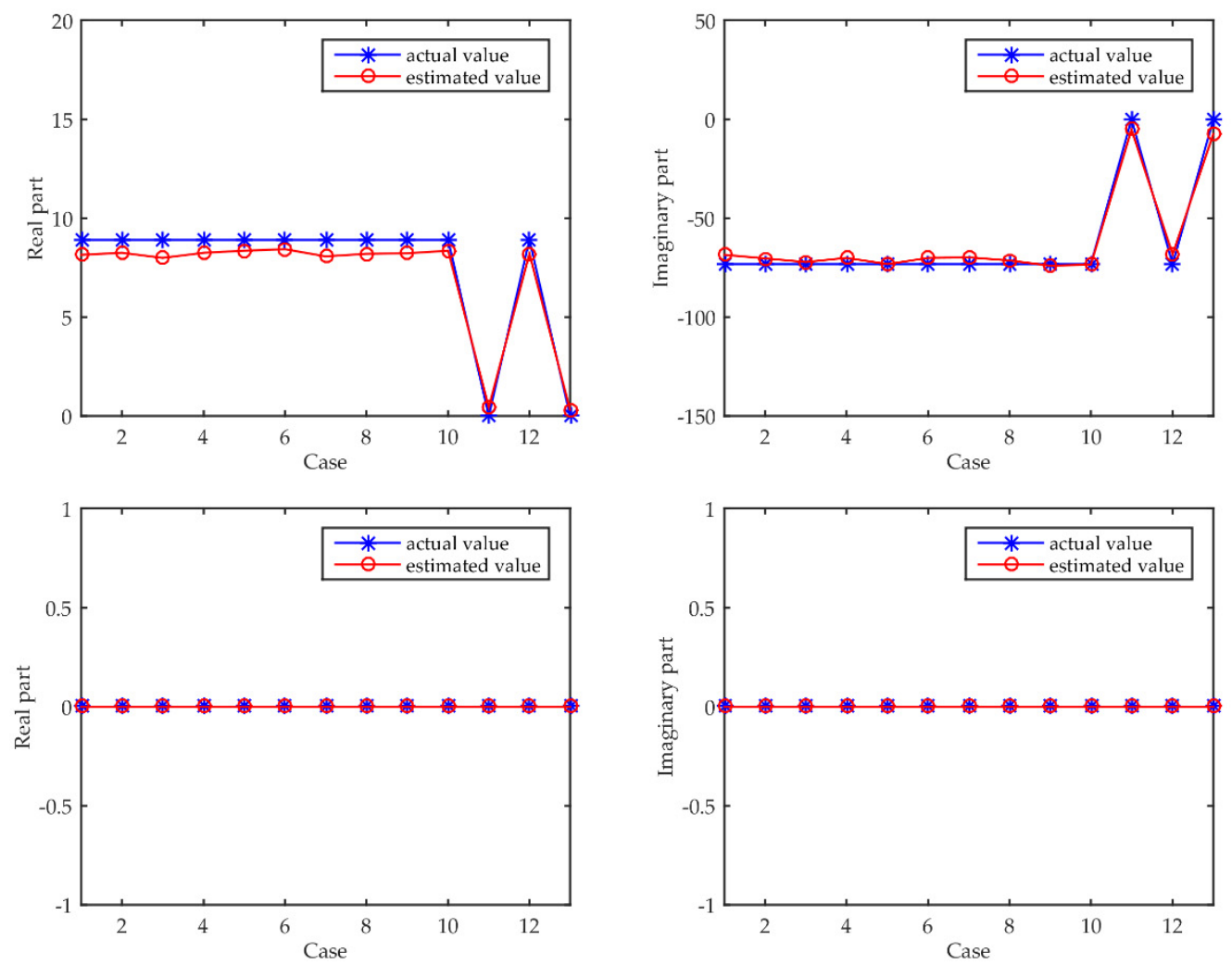

Figure 8 Admittance parameters of the SC model
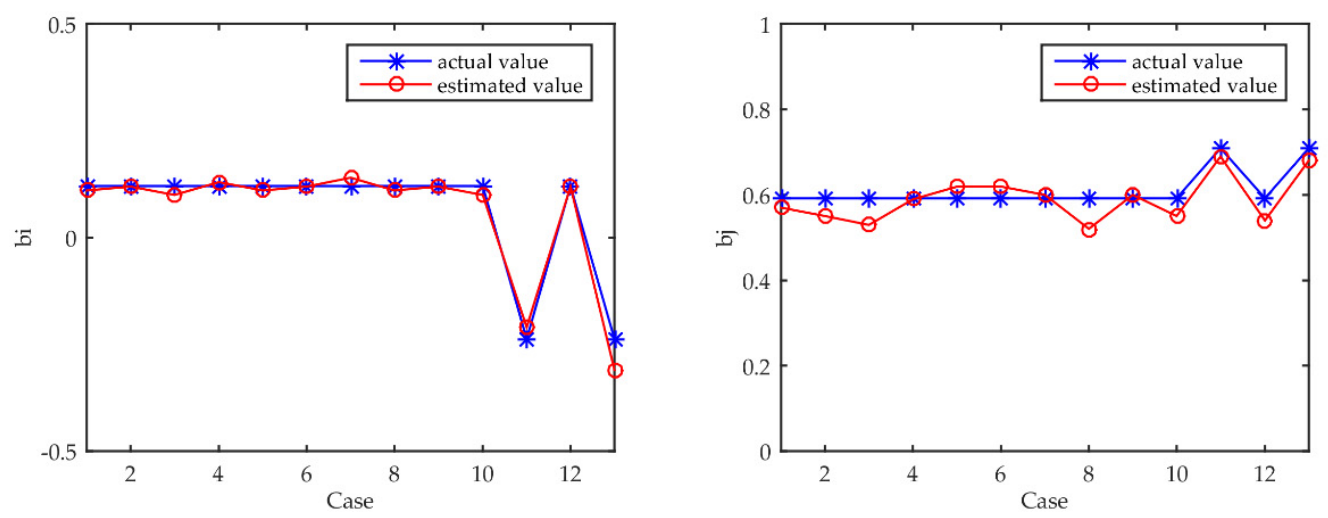

Figure 9 Susceptance parameters of the SC model

\section{Conclusions}

From the results of the calculation example, it can be seen that after the operating state of the interconnected power grid changes, the SC model also changes. The method proposed in this paper can update the SC model online without obtaining the operating parameters of the entire network, relying only on power big data, reducing online power flow calculation errors, and providing reliable data support for the reliable operation of the interconnected power grid. The rational use of data is of great significance. 


\section{Acknowledgments}

This research was funded by The National Key R\&D Plan of China 。

\section{References}

1. Huang, D.; Shu, Y.; Ruan, J.; Hu, Y., Ultra High Voltage Transmission in China: Developments, Current Status and Future Prospects. Proceedings of the IEEE 2009, 97, (3), 555-583

2. Liu, S.; Li, J.; Wu, J.; Guo, T.; Jiang, L., Ultra-high voltage/extra-high voltage transmission-line protection based on longitudinal tapped impedance. IET Generation, Transmission \& Distribution 2017, 11, (17), 4158-4166.

3. Rafique, S. F.; Shen, P.; Wang, Z.; Rafique, R.; Iqbal, T.; Ijaz, S.; Javaid, U., Global power grid interconnection for sustainable growth: concept, project and research direction. Iet Generation Transmission \& Distribution 2018, 12, (13), 31143123.

4. Zhang, F.; Xu, Z.; Jiao, B.; Yang, J.; Feng, J. In Global energy interconnection based optimal power systems planning - A global perspective 2016-2050, 2017 China International Electrical and Energy Conference (CIEEC), 25-27 Oct. 2017, 2017; 2017; pp 849-854

5. Mou, X.; Li, W.; Li, Z. In A preliminary study on the Thevenin equivalent impedance for power systems monitoring, 2011 4th International Conference on Electric Utility Deregulation and Restructuring and Power Technologies (DRPT), 6-9 July 2011, 2011; 2011; pp 730-733.

6. Deckmann, S.; Pizzolante, A.; Monticelli, A.; Stott, B.; Alsac, O., Studies on Power System Load Flow Equivalencing. IEEE Transactions on Power Apparatus and Systems 1980, PAS-99, (6), 23012310 .

7. Li, Z.; Yang, H. In Application of REI Equivalent in Reactive Power Optimization Control in Regional Power Grid, 2011 Asia-Pacific Power and Energy Engineering Conference, 25-28 March 2011, 2011; 2011; pp 1-4.

8. Yu, J.; Zhang, M.; Li, W. Y., Static Equivalent Method Based on Component Particularity Representation and Sensitivity Consistency. IEEE Trans. Power Syst. 2014, 29, (5), 2400-2408.

9. Fang, J. Overview of Big Data Development of Power Equipment Condition Monitoring. Power system protection and control. 2020, 48, (23), 176-186

10. Grigg, C.; Wong, P.; Albrecht, P.; Allan, R.; Bhavaraju, M.; Billinton, R.; Chen, Q.; Fong, C.; Haddad, S.; Kuruganty, S.; Li, W.; Mukerji, R.; Patton, D.; Rau, N.; Reppen, D.; Schneider, A.; Shahidehpour, M.; Singh, C., The IEEE Reliability Test System-1996. A report prepared by the Reliability Test System Task Force of the Application of Probability Methods Subcommittee. IEEE Trans.
Power Syst. 1999, 14, (3), 1010-1020. 